\title{
Proteomic profiling of plasma biomarkers in acute ischemic stroke due to large vessel occlusion
}

\author{
Chuan Qin ${ }^{1 \dagger}$, Xin-Ling Zhao ${ }^{1 \dagger}$, Xiao-Tong Ma ${ }^{1 \dagger}$, Luo-Qi Zhou' ${ }^{1}$, Long-jun Wu ${ }^{2}$, Ke Shang ${ }^{1}$, Wei Wang ${ }^{1}$ \\ and Dai-Shi Tian ${ }^{1 *}$
}

\begin{abstract}
Background: Acute ischemic stroke (AIS) due to large vessel occlusion (LVO) is a devastating cerebrovascular disorder, which could benefit from collateral circulation. Proteins associated with acute LVO pathogenesis and endothelial function may appear in blood samples of AIS patients due to LVO, thus permitting development of blood-based biomarkers for its diagnosis and prognosis.
\end{abstract}

Methods: This study is a single-center, retrospective, observational case-control trial. Consecutive patients who presented at the Department of Neurology of Tongji Hospital were recruited from July 2016 to April 2018. In the discovery phase, a proteomic approach with iTRAQ-based LC-MS/MS was used to investigate the altered proteomic pattern in plasma from patients with AIS due to LVO. In the validation study, Western blots was used to identify biomarkers associated with stroke diagnosis as well as their prognostic value associated with different collateral statuses.

Results: For this exploratory study, the proteomic analysis of plasma from 40 patients with AIS due to LVO and 20 healthy controls revealed seven differentially expressed proteins with a 1.2/0.83-fold or greater difference between groups. The four elevated proteins, PPBP (1.58 \pm 0.78 vs $0.98 \pm 0.37 ; P<0.001)$, THBS1 $(1.13 \pm 0.88$ vs $0.43 \pm 0.26$; $P<0.001)$, LYVE1 (1.61 \pm 0.55 vs $0.97 \pm 0.50 ; P<0.001)$, and IGF2 (1.19 \pm 0.42 vs $0.86 \pm 0.24 ; P<0.001$ ), were verified by Western blots analysis in an independent cohort including 33 patients and 33 controls. A strong interaction was observed between the four-protein panel and the diagnosis of AIS due to LVO (AUC 0.947; $P<0.001$ ). Furthermore, IGF2, LYVE1, and THBS1 were closely associated with collateral status (IGF2 0.115, 95\% Cl 0.016-0.841, P = 0.033; LYVE1 $0.183,95 \% \mathrm{Cl} 0.036-0.918, \mathrm{P}=0.039 ;$ THBS1 4.257, 95\% Cl 1.273-14.228, $\mathrm{P}=0.019)$, and proved to be independent predictors of good outcome (IGF2 0.115, 95\% Cl 0.015-0.866, P=0.036; LYVE1 0.028, 95\% Cl 0.002-0.334, P =0.005; THBS1 3.294, 95\% Cl 1.158-9.372, $\mathrm{P}=0.025)$ at a 3-month follow-up.

Conclusions: The identified 4-biomarker panel could provide diagnostic aid to the existing imaging modalities for AIS due to LVO, and the prognostic value of IGF2, LYVE1, and THBS1 was proved in predicting functional outcomes related to collateral status.

Trial registration ClinicalTrials.gov NCT 03122002. Retrospectively registered April 20, 2017. URL of trial registry record: https://www.clinicaltrials.gov/ct2/show/NCT03122002?term=NCT+03122002\&rank=1

Keywords: Ischemic stroke, Large vessel occlusion, Collateral circulation, Proteomics, Biomarkers

\footnotetext{
*Correspondence: tiandaishi@126.com

${ }^{\dagger}$ Chuan Qin, Xin-Ling Zhao and Xiao-Tong Ma contributed equally to the manuscript

${ }^{1}$ Department of Neurology, Tongji Hospital, Tongji Medical College, Huazhong University of Science and Technology, Wuhan 430030, People's Republic of China

Full list of author information is available at the end of the article
} 


\section{Background}

Large-vessel occlusions (LVOs) cause more than onethird of acutely presenting ischemic stroke events, and are associated with severe functional deficits [1]. Collateral circulation in patients with AIS may sustain the hypoperfused tissue at risk [2]. Good collaterals have been associated with improved recanalization, smaller infarct volume, and better clinical outcome [2]. We hypothesized that for patients with AIS due to LVO, related biomarkers with different collateral status could be potent predictors of functional outcomes at follow-up.

A novel quantitative proteomic technology, isobaric tagging for relative and absolute quantitation (iTRAQ), has recently become a powerful tool to characterize differentially expressed proteins and identify the biomarkers for central nervous systems disorders, particularly for stroke [3-5]. The new technology allows broad protein biomarker screenings, offering the hope to better understand the pathophysiological mechanisms underlying AIS due to LVO. Proteins associated with acute LVO pathogenesis and endothelial function may appear in blood samples of AIS patients due to LVO, thus permitting development of blood-based biomarkers for its diagnosis and prognosis.
In this study, we sought to discover potential biomarkers associated with AIS due to LVO by using proteomic technology with iTRAQ-based LC-MS/MS. Western blots analysis was used to further validate those candidate proteins for diagnosis of AIS due to LVO, and to evaluate their prognostic value related to different collaterals. This could potentially identify a proteomic signature that aids in understanding the pathophysiological pathway underlying the link between acute large vessel occlusion, different collateral status, and functional outcomes at follow-up.

\section{Methods}

\section{Study design and subjects}

An overview of the study design is shown in Fig. 1. Briefly, this study is a single-center, retrospective, observational case-control trial based on a prospectively collected stroke data base. Consecutive patients who presented at the Department of Neurology of Tongji Hospital were recruited from July 2016 to April 2018. The clinical diagnosis of acute ischemic stroke was confirmed by cranial magnetic resonance (MR) examinations with diffusion weighted imaging (DWI), and intracranial large vessel status was evaluated by digital subtraction

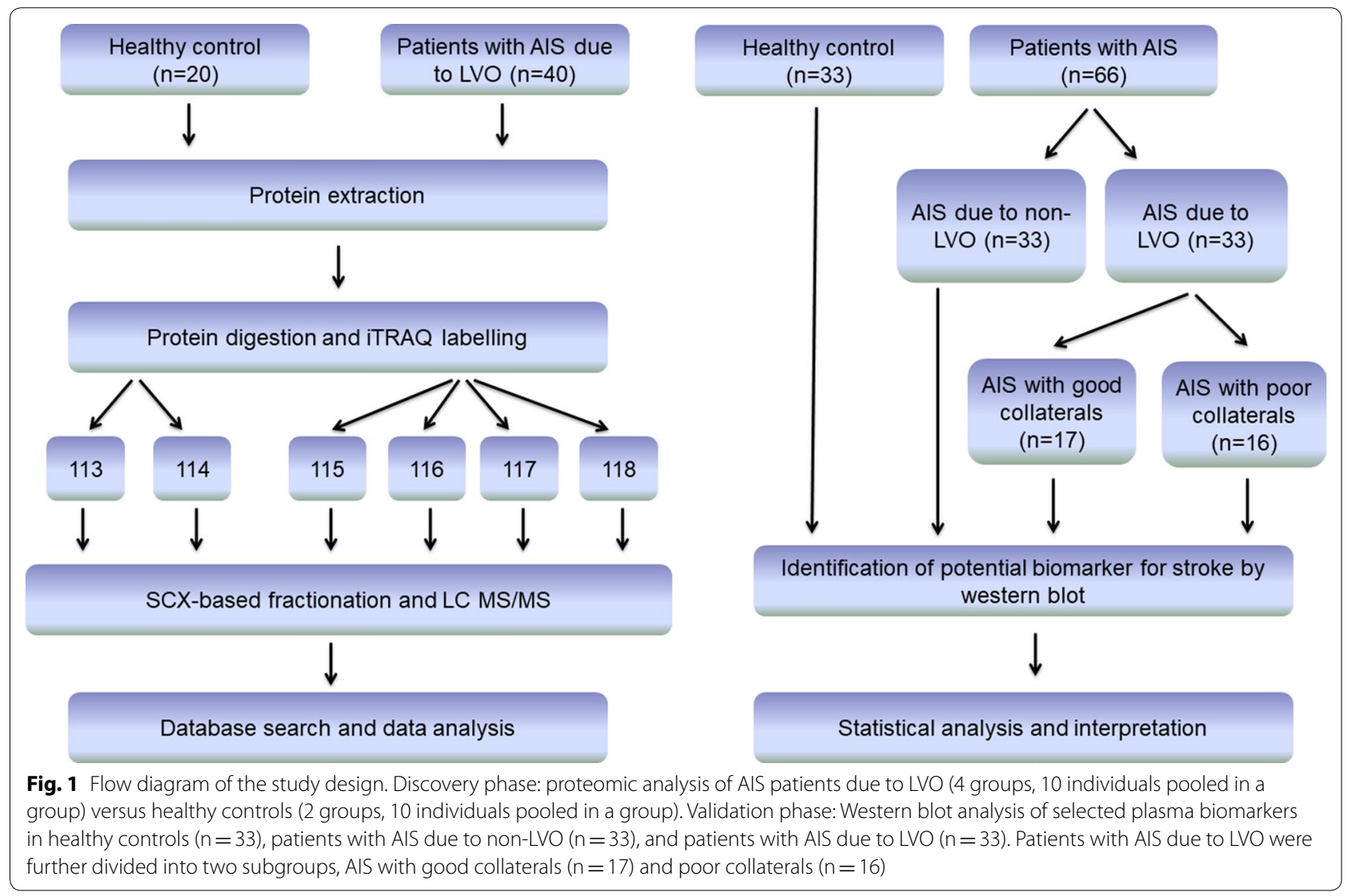


angiography (DSA) within 7 days in every patient. Studyspecific inclusion criteria were the presence of intracranial carotid artery terminus or first middle cerebral artery segment (M1) occlusion as confirmed on DSA, and sufficient display of the middle cerebral artery region. Studyspecific exclusion criteria were the administration of thrombolysis and thrombectomy, and baseline modified Rankin Scale $(\mathrm{mRS})>1$ before stroke onset. Patients with baseline mRS of $0-1$ who missed therapeutic time window or refused acute reperfusion therapy were enrolled to rule out the influence from baseline clinical differences and acute reperfusion therapy. Collateral status was not an entry criterion for the trial and was assessed using the American Society of Interventional and Therapeutic Neuroradiology/Society of Interventional Radiology (ASTIN/SIR) grading system by blinded observers. Healthy volunteers with no prior history of stroke were matched with patients with AIS due to LVO for age and sex, and were recruited as a healthy control group, to control for the confounding effects of additional risk factors. Cranial MR with DWI and angiography were performed in healthy volunteers to verify that no silent brain infarcts or artery stenosis were present. Patients with AIS in the middle cerebral artery region but no obstructive large vessels were matched with patients with AIS due to LVO for age and sex, and enrolled as AIS due to nonLVO group.

In the discovery phase, there were 20 volunteers in the healthy control group and 40 patients with stroke due to LVO in the AIS group. 10 individual samples of equal volume from each group were pooled together for the proteomics analysis. In the validation phase, another independent population that included 33 healthy controls, 33 patients with AIS due to non-LVO, and 33 patients with AIS due to LVO were enrolled for Western blots analysis. The patients with AIS due to LVO were further divided into two groups based on their ASTIN/ SIR grades: AIS with good collaterals (SGC, ASTIN/ SIR 3-4), and AIS with poor collaterals (SPC, ASTIN/ SIR 0-2). For prognostic prediction, patients with AIS due to LVO were divided into those with good prognosis (mRS 0-2) and those with poor prognosis (mRS 3-6) at a 3 -month follow-up. Table 1 provides demographic details of the study participants.

\section{Sample collection and preparation}

Blood samples were drawn from subjects within 7 days after stroke onset and the healthy controls, and collected in EDTA Vacutainer tubes (BD, Oxford, UK), immediately put on ice, centrifuged at $1280 \times g$ for $10 \mathrm{~min}$ at $4{ }^{\circ} \mathrm{C}$ to separate plasma within $1 \mathrm{~h}$, and stored at $-80^{\circ} \mathrm{C}$ until required.
iTRAQ-based quantitative proteomics analysis and Western blots validation

A detailed description of experimental procedures was provided in Additional file 1.

\section{Statistical analyses}

Statistical analyses were performed using SPSS 19.0 for Windows. The continuous variables were described by mean $\pm \mathrm{SD}$ or by medians and interquartile ranges (IQR), while categorical variables were described by percentages. Mann-Whitney U test, one-way analysis of variance (ANOVA) with Dunnett's post hoc test were used to compare differences between groups. Adjusted logistic regression models were used to calculate the odds ratio (OR) and 95\% confidence intervals (CI). Covariates included in the adjusted logistic regression models were determined by univariate logistic regression of every factor shown in Table 1. Logistic analysis and receiver operating characteristic (ROC) curves were employed to assess the diagnostic and prognostic accuracy of each biomarker. A combined ROC analysis was performed for panel diagnosis. All tests were considered statistically significant at $\mathrm{P}<0.05$.

\section{Results}

\section{Clinical characteristics of patients}

Baseline characteristic details of the patients and controls participating in the study are summarized in Table 1 . For the discovery phase study, 40 patients with AIS due to LVO attending our hospital between July 2016 and April 2017 and 20 healthy control subjects were enrolled (Fig. 1). Compared with healthy controls, patients with AIS due to LVO had higher systolic blood pressure and homocysteinemia ( $\mathrm{HCY}$ ) levels. There were no significant differences in age, sex, medical history, or laboratory data between the two groups (Table 1).

For the validation phase study, 33 healthy controls, 33 patients with AIS due to non-LVO and 33 patients with AIS due to LVO were prospectively enrolled from April 2017 to April 2018 (Fig. 1). Higher systolic and diastolic blood pressure, higher HCY levels, higher hypertension and diabetes mellitus rates, as well as more patients with smoking history, were found in the AIS group than in the control group. Patients with AIS were divided into two subgroups: 33 patients with AIS due to LVO and 33 patients with AIS due to non-LVO. Lower lowdensity lipoprotein level was found in the LVO group. Patients with AIS due to LVO were further divided into two subgroups based on their ASTIN/SIR grades: 17 patients with good collaterals (SGC) and 16 patients with poor collaterals (SPC). There were no significant 


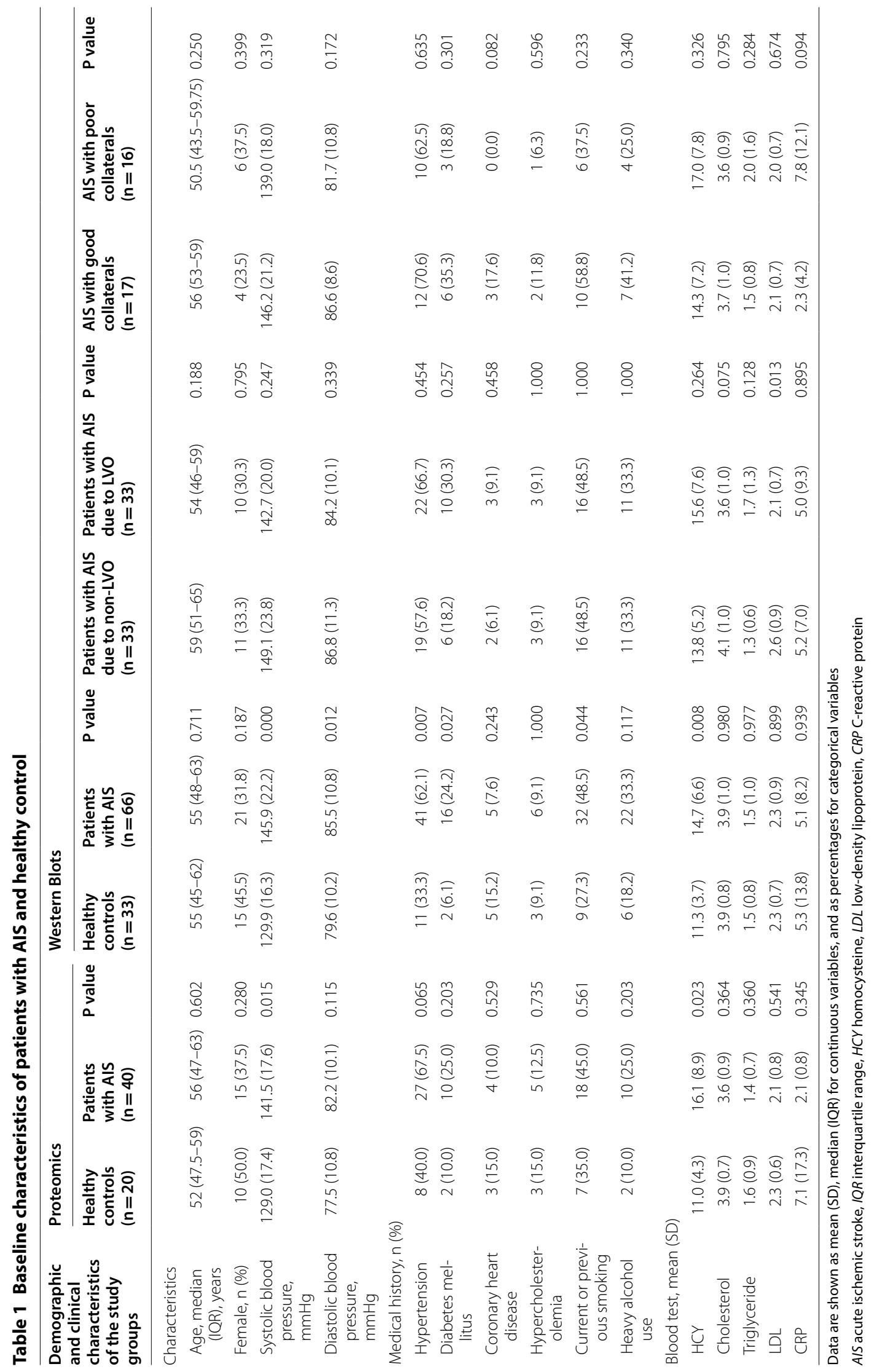


differences in demographic characteristics or laboratory data between the SGC and SPC groups (Table 1).

\section{Quantitative proteomic analysis reveals a specific proteomic profile in AIS due to LVO}

We first compared plasma protein profiles from 40 consecutive patients with AIS due to LVO and 20 healthy controls. Pooled plasma samples were analyzed in replicates using 6-plex iTRAQ with LC-MS/MS. Patients with AIS due to LVO had a specific proteomic signature. The molecular features that were detected are depicted in the heatmap (Additional file 1: Figures S1 and S2). Seven differentially expressed proteins, thrombospondin 1 (THBS1), secreted phosphoprotein 2 (SPP2), lymphatic vessel endothelial hyaluronic acid receptor 1 (LYVE1), cadherin 1 (CDH1), insulin-like growth factor 2 (IGF2), pro-platelet basic protein (PPBP), and apolipoprotein $\mathrm{C}$ 4-2 (APOC4-APOC2), were finally acquired with a 1.2/0.83-fold or greater difference in abundance in AIS samples compared to healthy controls (Additional file 1: Table S1). Of these seven proteins, THBS1, LYVE1, IGF2, and PPBP were more abundant in AIS due to LVO samples, while SPP2, CDH1, and APOC4-APOC2 were downregulated compared to control samples.

\section{Validation of potential biomarkers by Western blots} analysis

Interestingly, among these differentially expressed proteins identified in plasma samples of AIS due to LVO, all the upregulated proteins, IGF2, LYVE1, PPBP, and THBS1, are known to be involved in regulating blood hemostasis and endothelial function [6-11]. We then verified the differential abundance of four selected proteins (IGF2, LYVE1, PPBP, and THBS1) using Western blots analysis. Consistent with the tendency found in proteomics, Western blots results showed similar alterations in an independent population, as the levels of those four identified proteins were significantly higher in the AIS due to LVO group compared to the healthy control group (Fig. 2, Tables 2 and 3). To describe the specificity for AIS due to LVO, we then detected the levels of the four identified proteins in patients with AIS due to non-LVO. Interestingly, expression of IGF2, LYVE1 and PPBP showed no significant difference between AIS due to non-LVO and healthy control, except that THBS1 was higher in AIS due to non-LVO group. Further, comparisons between the non-LVO and LVO groups presented higher expression of IGF2, LYVE1, PPBP and THBS1 in the LVO group (Fig. 2,

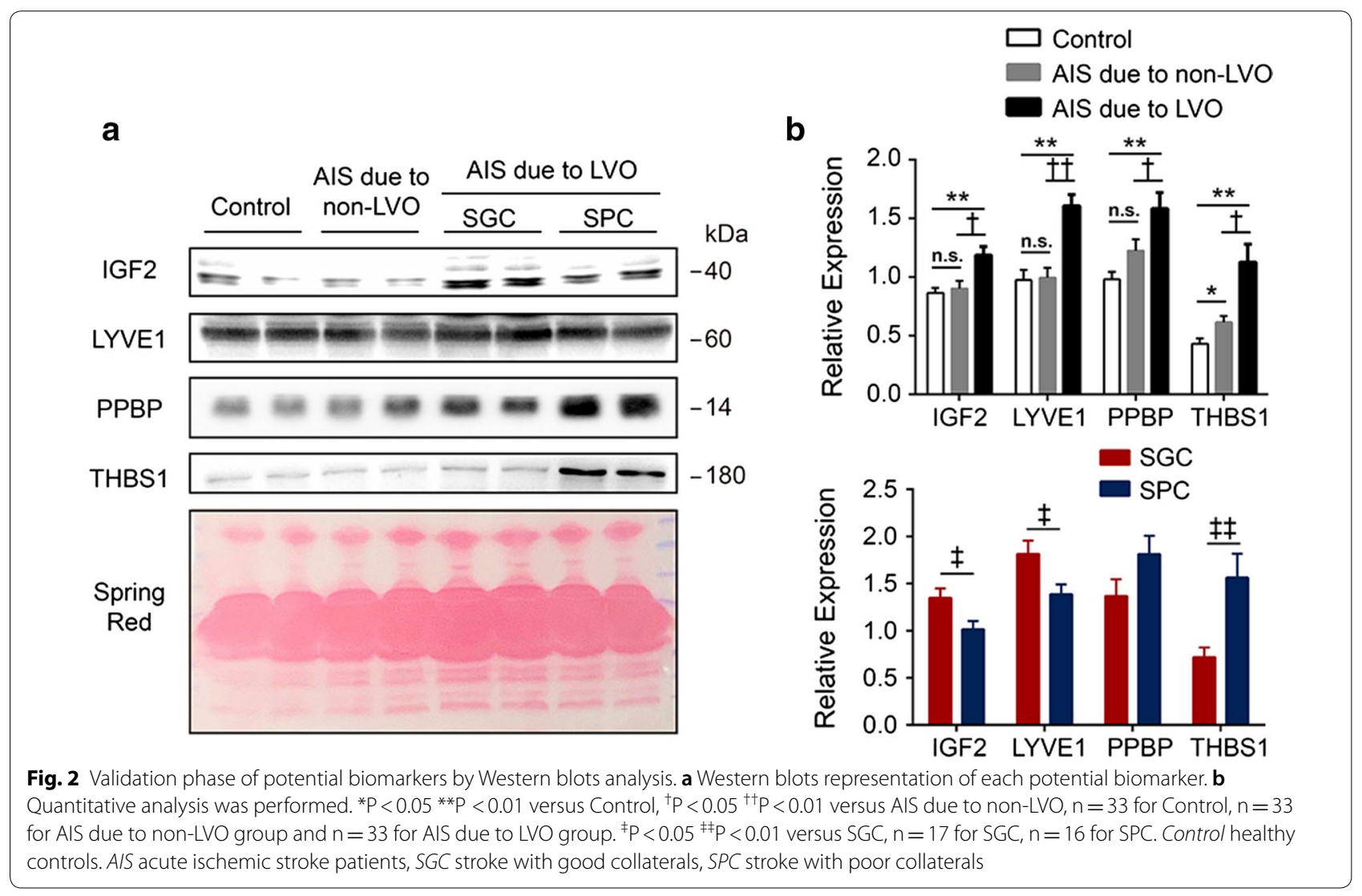


Table 2). Altered levels of these proteins might indicate endothelial dysfunction and clinical implications of large vessel occlusion.

Patients with AIS due to LVO were further divided into SGC and SPC subgroups, according to the ASTIN/ SIR grades assigned to their collaterals. Comparisons between the SGC and SPC group indicated higher expression of LYVE1 and IGF2 in the SGC group and higher level of THBS1 in the SPC group, but no significant difference in PPBP (Fig. 2, Table 4).

\section{Predictive potential of biomarkers}

In order to define the capacity of these potential biomarkers for diagnosis, logistic regression and receiver operating characteristic (ROC) curves were employed. Logistic analysis results supported the role of plasma levels of IGF2, LYVE1, PPBP, and THBS1 as potential biomarkers that indicate a significantly increased risk of AIS due to LVO (Table 3).

We then assessed the diagnostic efficiency of the potential biomarkers using ROC analysis. The areas under the

Table 2 Plasma levels of each biomarker in different groups

\begin{tabular}{|c|c|c|c|c|c|c|c|}
\hline Proteins & $\begin{array}{l}\text { Healthy controls } \\
(n=33)\end{array}$ & $\begin{array}{l}\text { Patients with AIS due } \\
\text { to non-LVO }(n=33)\end{array}$ & $\begin{array}{l}\text { Patients with AIS due } \\
\text { to LVO }(n=33)\end{array}$ & $P$ value & $\mathrm{P}^{\mathrm{a}}$ value & $\mathrm{P}^{\mathbf{b}}$ value & $P^{c}$ value \\
\hline IGF2 & $0.86 \pm 0.24$ & $0.90 \pm 0.50$ & $1.19 \pm 0.42$ & 0.001 & 0.948 & 0.001 & 0.017 \\
\hline LYVE1 & $0.97 \pm 0.50$ & $0.99 \pm 0.49$ & $1.61 \pm 0.56$ & $<0.001$ & 0.998 & $<0.001$ & $<0.001$ \\
\hline PPBP & $0.98 \pm 0.37$ & $1.23 \pm 0.55$ & $1.58 \pm 0.78$ & $<0.001$ & 0.081 & 0.001 & 0.045 \\
\hline THBS1 & $0.43 \pm 0.26$ & $0.62 \pm 0.29$ & $1.13 \pm 0.88$ & $<0.001$ & 0.030 & $<0.001$ & 0.010 \\
\hline
\end{tabular}

Data are shown as mean $\pm S D$. One-way analysis of variance (ANOVA) with Dunnett's post hoc test

AIS acute ischemic stroke, LVO large vessel occlusion

a Patients with AIS due to non-LVO versus healthy controls

b Patients with AIS due to LVO versus healthy controls

c Patients with AIS due to LVO versus patients with AIS due to non-LVO

Table 3 Correlation analysis of each biomarker with diagnosis

\begin{tabular}{|c|c|c|c|c|c|c|}
\hline Proteins & $\mathrm{OR}^{\mathrm{a}}\left(95 \% \mathrm{Cl}^{\mathrm{a}}\right)$ & $P^{a}$ value & $\mathrm{OR}^{\mathrm{b}}\left(95 \% \mathrm{Cl}^{\mathrm{b}}\right)$ & $\mathrm{P}^{\mathbf{b}}$ value & AUC $(95 \% \mathrm{Cl})$ & $P^{c}$ value \\
\hline IGF2 & $17.365(2.862-105.358)$ & 0.002 & $47.564(5.133-440.757)$ & 0.001 & $0.731(0.609-0.853)$ & 0.001 \\
\hline LYVE1 & $10.320(2.986-35.669)$ & $<0.001$ & 7.707 (2.215-26.813) & 0.001 & $0.813(0.707-0.919)$ & $<0.001$ \\
\hline PPBP & $7.682(2.201-26.813)$ & 0.001 & $8.163(1.903-35.014)$ & 0.005 & $0.754(0.638-0.870)$ & $<0.001$ \\
\hline THBS1 & $13.282(2.630-67.079)$ & 0.002 & $14.132(2.401-83.185)$ & 0.003 & $0.769(0.652-0.885)$ & $<0.001$ \\
\hline
\end{tabular}

AUC area under the receiver operating characteristic curve, OR odds ratio, Cl confidence interval, IGF2 insulin like growth factor 2 , LYVE1 lymphatic vessel endothelial hyaluronan receptor 1, PPBP pro-platelet basic protein, THBS1 thrombospondin 1

a Logistic regression analysis, OR and $95 \% \mathrm{Cl}$ without adjusting

b Logistic regression analysis, OR and $95 \% \mathrm{Cl}$ with adjusting for age, gender, blood pressure, medical history and blood test

c Receiver operating characteristic curve analysis

Table 4 Correlation analysis of each biomarker with prognosis in AIS with different collaterals

\begin{tabular}{|c|c|c|c|c|c|c|c|}
\hline Proteins & $\begin{array}{l}\text { AIS with good } \\
\text { collaterals }(n=17)\end{array}$ & $\begin{array}{l}\text { AIS with poor } \\
\text { collaterals }(n=16)\end{array}$ & $P$ value & $\mathrm{OR}^{\mathrm{a}}\left(95 \% \mathrm{Cl}^{\mathrm{a}}\right)$ & $\mathrm{P}^{\mathrm{a}}$ value & $O R^{b}\left(95 \% \mathrm{Cl}^{b}\right)$ & $\mathrm{P}^{\mathrm{b}}$ value \\
\hline IGF2 & $1.35 \pm 0.41$ & $1.02 \pm 0.35$ & 0.022 & $0.115(0.016-0.841)$ & 0.033 & $0.115(0.015-0.866)$ & 0.036 \\
\hline LYVE1 & $1.82 \pm 0.58$ & $1.39 \pm 0.42$ & 0.026 & $0.183(0.036-0.918)$ & 0.039 & $0.028(0.002-0.334)$ & 0.005 \\
\hline PPBP & $1.37 \pm 0.73$ & $1.81 \pm 0.77$ & 0.111 & $1.939(0.707-5.321)$ & 0.198 & $2.806(0.933-8.439)$ & 0.066 \\
\hline THBS1 & $0.72 \pm 0.42$ & $1.56 \pm 1.02$ & 0.005 & $4.257(1.273-14.228)$ & 0.019 & $3.294(1.158-9.372)$ & 0.025 \\
\hline
\end{tabular}

Data are shown as mean \pm SD

OR odds ratio, Cl confidence interval, IGF2 insulin like growth factor 2, LYVE1 lymphatic vessel endothelial hyaluronan receptor 1, PPBP pro-platelet basic protein, THBS1 thrombospondin 1

a Logistic regression analysis of each biomarker with different collaterals, OR and $95 \% \mathrm{Cl}$ with adjusting for age, gender, blood pressure, medical history and blood test

b Logistic regression analysis each biomarker with prognosis, OR and $95 \% \mathrm{Cl}$ with adjusting for age, gender, blood pressure, medical history and blood test 
ROC curve (AUC) were $0.731(0.609-0.853)$ for IGF2, 0.813 (0.707-0.919) for LYVE1, $0.754(0.638-0.870)$ for PPBP, and $0.769(0.652-0.885)$ for THBS1, respectively (Fig. 3a, Table 3). Furthermore, the diagnostic potential of the 4-protein panel was also evaluated. The AUC value of the panel reached 0.947 for distinguishing patients with AIS due to LVO from healthy individuals, which was interpreted as excellent classification performance (Fig. 3b).

We further investigated the association between plasma levels of these identified potential biomarkers and the prognosis of AIS due to LVO. Patients were divided into those with good prognosis (modified Rankin scale, mRS 0-2) and those with poor prognosis (mRS 3-6) at a 3-month follow-up. Logistic analysis results showed that higher IGF2 and LYVE1 levels predicted favorable outcomes, while higher THBS1 levels were related to a poor prognosis. The results implicated that the three collateral-related proteins (IGF2, LYVE1, and THBS1) were prognostic for patients with AIS due to LVO, while PPBP showed no potential for outcome prediction (Table 4).

\section{Discussion}

The aim of this study was to analyze plasma proteomic profiles of patients with AIS due to LVO, and to identify potential biomarkers that are associated with the status of collateral circulation. This study yielded two main findings. First, four upregulated proteins, IGF2, LYVE1, PPBP, and THBS1, were identified in patients with AIS due to LVO, compared to healthy controls and patients with AIS due to non-LVO. Second, IGF2, LYVE1, and THBS1 were closely associated with collateral status, and were shown to be independent predictors of favorable outcome at a 3-month follow-up.

Insulin-like growth factor 2 (IGF2) was found to be required for angiogenesis in growing tumors via the induction of hypoxia-related vascular endothelial growth factor [7]. Although no much evidence in the association between IGF2 and the risk of ischemic stroke yet, IGF2 might contribute to vascular remodeling and neovascularization by enhancing the recruitment and incorporation of endothelial progenitor cells into areas of ischemic injury [7].

Lymphatic vessel endothelial hyaluronic acid receptor 1 (LYVE1), a cell surface receptor on lymphatic endothelial cells, is involved in capillary sprouting through lymphatic/blood endothelial cell interactions [8]. LYVE1 levels have been reported significantly increased in patients with malignant middle cerebral artery infarction, and indicated as a potential marker of early prediction [12].

Pro-platelet basic protein (PPBP) can be released in large amounts from activated platelets [13], and is a key participant in both homeostasis and inflammation during ischemia $[9,10]$. PPBP has been identified as an inflammatory biomarker increased in central retinal vein ischemic occlusion [9], and as a potential risk factor of coronary heart disease in patients with hyperlipidaemia [10].

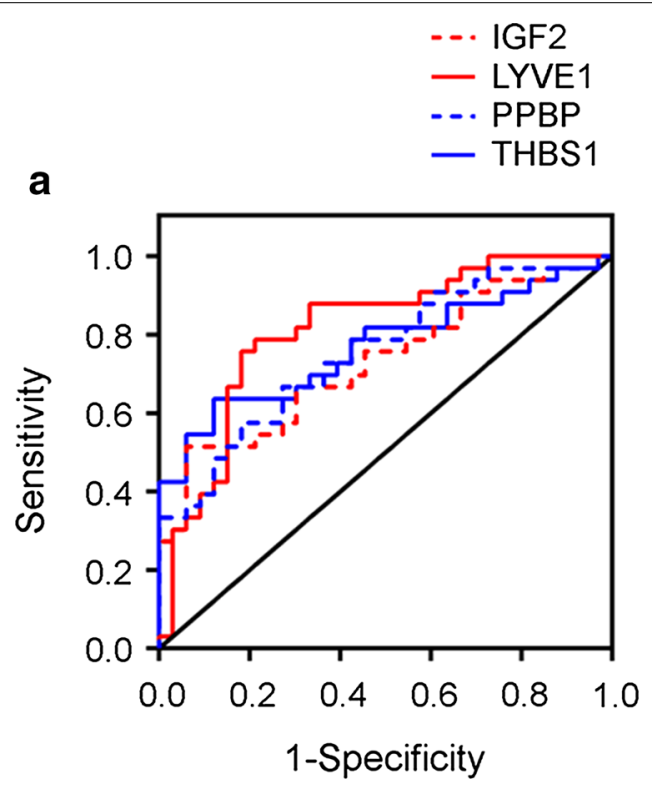

b
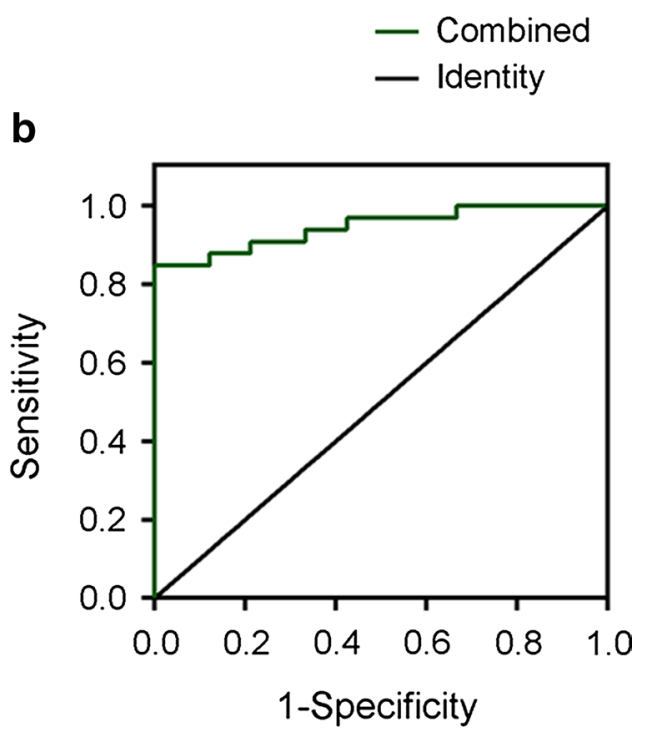

Fig. 3 Diagnostic efficiency of the potential biomarkers. a Receiver operating characteristic curve of biomarkers in diagnosis of AIS due to LVO. IGF2, LYVE1, PPBP and THBS1 could be efficient in diagnosis, and $\mathbf{b}$ 4-protein panel combined has better performance 
Thrombospondin 1 (THBS1) was first isolated from human blood platelets as a thrombin-sensitive protein [14]. THBS1 is a potent regulator of angiogenesis that functions to concurrently inhibit endothelial cell migration and the release of vascular endothelial growth factor from the extracellular matrix [11]. Cerebral THBS1 expression is upregulated in experimental focal cerebral ischemia [15] and the circulating THBS1 were elevated in patients with ischemic stroke [14].

The proteomic signature of patients with AIS due to LVO in our study is characterized by the increase in these four proteins, when compared to both healthy controls and AIS due to non-LVO group. Large vessel occlusion may explain the change, as the four proteins are involved in regulating blood hemostasis and endothelial function. The combination of the four biomarkers could robustly identify (with an accuracy $>90 \%$ ) these patients, offering excellent predictive values for distinguishing acute LVO strokes. Blood is easy to obtain, making this biomarker panel a useful predictor that could be screened for in large-scale clinical trials and for future clinical use.

Collateral circulation refers to the network of blood vessels that contribute towards maintaining blood flow in the absence of the primary conduit [16]. The degree of such collateral blood supply varies amongst individuals and can influence outcomes, especially in patients with AIS due to LVO [16]. Patients with good collateral flow demonstrated less hypoperfused tissue, less infarct growth within the penumbra zone, and smaller infarct volumes with better outcomes than those with poor collaterals [17]. Our study found that elevated IGF2 and LYVE1 levels and lower THBS1 levels were associated with better collateral circulation in patients with AIS due to LVO. No significant difference was observed in PPBP. Interestingly, the three collateral-related proteins (IGF2, LYVE1, and THBS1) were prognostic, as patients with relatively higher IGF2 and LYVE1 levels and lower THBS1 levels were more likely to have good outcomes, while PPBP showed no potential as a predictor of outcomes. IGF2 and LYVE1 are key determinants in vascular remodeling and neovascularization [6-8], and THBS1 has been shown to inhibit angiogenesis [11]. Despite the relationship between endothelium function and collateral circulation is complex, our findings suggest that IGF2, LYVE1, and THBS1 could be associated with cerebral collateral circulation and thus clinical outcomes after AIS due to LVO, making them potential predictors.

\section{Limitations}

The present study was subject to several limitations. The small sample size and the retrospective nature of the study indicate that biases are inevitable. Moreover, all patients were ethnically Chinese; our results may not apply to other populations. Further research on the dynamic changes of the four biomarkers from hyperacute phase to chronic phase of ischemic stroke is needed to elucidate details concerning the diagnostic and prognostic values of these biomarkers in stroke. The development of clinical trials in large scale population and long term assessment is also warranted.

\section{Conclusions}

To our knowledge, this is the first study of a blood-based biomarker panel with high accuracy for detecting AIS due to LVO. Although actual mechanisms of collateral blood flow regulation remain elusive, the collateralrelated proteins found here showed potential as predictors of clinical outcomes of patients with AIS due to LVO at a 3-month follow-up. Both the diagnostic biomarker panel and the prognostic predictors require external validation in a more diverse demographic group with longer follow-up assessment before further development for clinical use.

\section{Additional file}

Additional file 1. Proteomic profiling of plasma biomarkers in acute ischemic stroke due to large vessel occlusion.

\section{Abbreviations \\ AIS: acute ischemic stroke; LVO: large vessel occlusions; SGC: AIS with good collaterals; SPC: AIS with poor collaterals; DSA: digital subtraction angiography; ROC: receiver operating characteristic; AUC: areas under the ROC curve; OR: odds ratio; Cl: confidence interval; IGF2: insulin like growth factor 2; LYVE1: lymphatic vessel endothelial hyaluronan receptor 1; PPBP: pro-platelet basic protein; THBS1: thrombospondin 1.}

\section{Acknowledgements}

Not applicable.

\section{Authors' contributions}

CQ, DST, and WW designed the study and contributed to aspects related to the cohort design; XLZ and XTM acquired data and analyzed data; XLZ performed the proteomics analysis; XTM performed the Western blots analysis; LQZ, and KS performed statistical analysis; CQ, XLZ and DST drafted the manuscript. All authors revised the manuscript critically for important intellectual content. All authors are accountable for all aspects of the manuscript. All authors read and approved the final manuscript.

\section{Funding}

This work was supported by National Natural Science Foundation of China (61327902-6, 91332108 to W. Wang, 81571132,81873743 to D.S. Tian, 81801223 to C. Qin).

\section{Availability of data and materials}

The datasets used and analysed during the current study are available from the corresponding author on reasonable request.

\section{Ethics approval and consent to participate}

This study was approved by the Ethics Committee of Tongji Medical College, Huazhong University of Science and Technology, Wuhan, China (No. 
S022/2017). All the procedures followed were in accordance with the Helsinki Declaration 2000.

\section{Consent for publication}

Written informed consents were obtained from all patients or their authorizer.

\section{Competing interests}

The authors declare that they have no competing interests.

\section{Author details}

1 Department of Neurology, Tongji Hospital, Tongji Medical College, Huazhong University of Science and Technology, Wuhan 430030, People's Republic of China. ${ }^{2}$ Department of Neurology, Mayo Clinic, Rochester, MN 55905, USA.

Received: 8 May 2019 Accepted: 25 June 2019

Published online: 01 July 2019

\section{References}

1. Rocha M, Jovin TG. Fast versus slow progressors of infarct growth in large vessel occlusion stroke: clinical and research implications. Stroke. 2017;48:2621-7.

2. Kawano H, Bivard A, Lin L, Spratt NJ, Miteff F, Parsons MW, Levi CR. Relationship between collateral status, contrast transit, and contrast density in acute ischemic stroke. Stroke. 2016;47:742-9.

3. Sharma R, Gowda H, Chavan S, Advani J, Kelkar D, Kumar GS, Bhattacharjee $\mathrm{M}$, Chaerkady $\mathrm{R}$, Prasad TS, Pandey $\mathrm{A}$, et al. Proteomic signature of endothelial dysfunction identified in the serum of acute ischemic stroke patients by the iTRAQ-based LC-MS approach. J Proteome Res. 2015;14:2466-79.

4. Laborde CM, Mourino-Alvarez L, Akerstrom F, Padial LR, Vivanco F, GilDones F, Barderas MG. Potential blood biomarkers for stroke. Expert Rev Proteomics. 2012;9:437-49.

5. Walsh KB, Hart K, Roll S, Sperling M, Unruh D, Davidson WS, Lindsell CJ Adeoye $\mathrm{O}$. Apolipoprotein A-I and paraoxonase-1 are potential blood biomarkers for ischemic stroke diagnosis. J Stroke Cerebrovasc Dis. 2016;25:1360-5.

6. Zaina S, Pettersson L, Thomsen AB, Chai CM, Qi Z, Thyberg J, Nilsson J. Shortened life span, bradycardia, and hypotension in mice with targeted expression of an lgf2 transgene in smooth muscle cells. Endocrinology. 2003;144:2695-703.
7. Maeng YS, Choi HJ, Kwon JY, Park YW, Choi KS, Min JK, Kim YH, Suh PG, Kang KS, Won MH, et al. Endothelial progenitor cell homing: prominent role of the IGF2-IGF2R-PLCbeta2 axis. Blood. 2009;113:233-43.

8. Stapor PC, Azimi MS, Ahsan T, Murfee WL. An angiogenesis model for investigating multicellular interactions across intact microvascular networks. Am J Physiol Heart Circ Physiol. 2013;304:H235-45.

9. Ehlken C, Grundel B, Michels D, Junker B, Stahl A, Schlunck G, Hansen LL, Feltgen N, Martin G, Agostini HT, Pielen A. Increased expression of angiogenic and inflammatory proteins in the vitreous of patients with ischemic central retinal vein occlusion. PLoS ONE. 2015;10:e0126859.

10. Maneerat Y, Prasongsukarn K, Benjathummarak S, Dechkhajorn W. PPBP and DEFA1/DEFA3 genes in hyperlipidaemia as feasible synergistic inflammatory biomarkers for coronary heart disease. Lipids Health Dis. 2017;16:80.

11. Yamauchi M, Imajoh-Ohmi S, Shibuya M. Novel antiangiogenic pathway of thrombospondin-1 mediated by suppression of the cell cycle. Cancer Sci. 2007;98:1491-7.

12. Xia C, Li XQ, Zhou ZH, Chen HS. Identification of cytokines for early prediction of malignant middle cerebral artery infarction. Int J Neurosci. 2017;127:86-91.

13. Stankiewicz AM, Goscik J, Swiergiel AH, Majewska A, Wieczorek M, Juszczak GR, Lisowski P. Social stress increases expression of hemoglobin genes in mouse prefrontal cortex. BMC Neurosci. 2014;15:130.

14. Gao JB, Tang WD, Wang HX, Xu Y. Predictive value of thrombospondin-1 for outcomes in patients with acute ischemic stroke. Clin Chim Acta. 2015;450:176-80.

15. Lin TN, Kim GM, Chen JJ, Cheung WM, He YY, Hsu CY. Differential regulation of thrombospondin-1 and thrombospondin-2 after focal cerebral ischemia/reperfusion. Stroke. 2003;34:177-86.

16. Gill D. Cerebral collateral circulation and acute ischaemic stroke. Eur J Neurol. 2016:23:1696-7.

17. Tan BY, Wan-Yee K, Paliwal P, Gopinathan A, Nadarajah M, Ting E, Venketasubramanian N, Seet RC, Chan BP, Teoh HL, et al. Good intracranial collaterals trump poor ASPECTS (Alberta Stroke Program Early CT Score) for intravenous thrombolysis in anterior circulation acute ischemic stroke. Stroke. 2016:47:2292-8.

\section{Publisher's Note}

Springer Nature remains neutral with regard to jurisdictional claims in published maps and institutional affiliations.
Ready to submit your research? Choose BMC and benefit from:

- fast, convenient online submission

- thorough peer review by experienced researchers in your field

- rapid publication on acceptance

- support for research data, including large and complex data types

- gold Open Access which fosters wider collaboration and increased citations

- maximum visibility for your research: over 100M website views per year

At BMC, research is always in progress.

Learn more biomedcentral.com/submissions 